\title{
Local Impurity Phase Pinning and Pinning Force in Charge Density Waves
}

\author{
A. Kobelkov, F. Gleisberg \\ and W. Wonneberger \\ Department of Physics \\ University of Ulm \\ D 89069 Ulm/Germany
}

\begin{abstract}
Starting from the static Fukuyama-Lee-Rice equation for a threedimensional incommensurate charge density wave (CDW) in quasi onedimensional conductors a solvable model for local phase pinning by impurities is defined and studied. We find that average CDW energy and average pinning force show critical behaviour with respect to the pinning parameter $h$. Specifically the pinning force exhibits a threshold at $h=1$ with exponent $\beta=2$. Our model examplifies a general concept of local impurity pinning in which the force exerted by the impurity on the periodic CDW structure becomes multivalued and metastable states appear beyond a threshold. It is found that local impurity pinning becomes less effective at low temperatures and may eventually cease completely. These results are independent of spatial dimensionality as expected for local impurity pinning. Comparison with Larkin's model is also made.
\end{abstract}

PACS: 71.45.Lr, 71.55.Jv, 72.15.Nj

\section{Introduction}

In charge density waves (CDW) which appear below the Peierls transition temperature in quasi one-dimensional metals [1], pinning of the order parameter phase at point defects is an important effect. Phase pinning results from the electrostatic coupling between the spatially periodic charge modulation in the CDW and the electric potential of an impurity. The phase $\varphi$ determines the position of the CDW with respect to the host lattice. The energy and force contributions of an individual impurity become periodic functions of $\varphi$. Distortions of the phase near the impurity produce a positive elastic energy. By properly adjusting the phase pattern near the impurity a net pinning force can be possible. Phase pinning in 
CDW is not fully understood at present. Well known is the weak or collective pinning limit. In this case each impurity only slightly distorts the local phase, however, many impurities act coherently in a macroscopic metastable Lee-Rice domain [2, 3] and produce a small pinning force. This mechanism has its analogue in pinning of flux lines in type II superconductors [4, 5]. The opposite limit of strong pinning is obtained when the pinning strength of a single impurity is so large that the elastic energy of local phase deformations is neglegible [2]. Intermediate concepts have also been explored [6, 7, 8].

In this paper we point out a number of special features for local phase pinning by impurities. Local phase pinning describes pinning effects linear in the concentration of impurities. We study a three-dimensional incommensurate CDW within a solvable model. We find that the averaged CDW energy shows a weak singularity as function of the pinning parameter $h$ at $h=1$ characterized by an exponent $b$ which is calculated. The formal limit of strong pinning is reached only for very large $h$. The pinning force has a threshold at $h=1$, i.e., it vanishes for $h \leq 1$ and then starts with a "critical" exponent similar to a phase transition. Our model realizes a general concept of local impurity pinning when the force exerted by the impurity on the periodic structure (CDW in our case) becomes multivalued and metastable states appear [5]. In a single chain model a similar problem has been considered recently in [9, 13] neglecting the screening.

Larkin's model [9] has been extended in [10] to include dynamics. It has meanwhile evolved into a complete concept of CDW and SDW dynamics [11, 12]. Here, we restrict ourselves to the static properties of a complementary model.

The model is introduced in Sec. 2 . It is exactly solved for average energy (Sec. 3) and average pinning force (Sec. 4) in the screened limit when an abundance of quasi-particles eliminates Coulomb forces. The latter act between the local charges that are produced by phase deformations in CDW. In Sec. 5 we discuss descreening in semiconducting CDW. We show that in lowering the temperature local impurity pinning becomes less and less effective and may even cease completely. Finally Sec. 6 gives a detailed account of Larkin's model [9] and its relation to our results.

\section{Pinning Model}

Following [3] we write the phase dependent part of the static energy density of incommensurate CDW plus one impurity at position $\mathbf{x}_{i}$ as

$\mathcal{H}=\frac{1}{2} K\left\{\left(\frac{\partial \varphi}{\partial z}\right)^{2}+\frac{v_{t 1}^{2}}{v_{F}^{2}}\left(\frac{\partial \varphi}{\partial x}\right)^{2}+\frac{v_{t 2}^{2}}{v_{F}^{2}}\left(\frac{\partial \varphi}{\partial y}\right)^{2}\right\}+V_{0} \delta\left(\mathbf{x}-\mathbf{x}_{i}\right) \cos \left(\mathbf{Q} \mathbf{x}_{i}+\varphi(\mathbf{x})\right)$

The stiffness constant in the fully screened limit and for a rectangular lattice is 


$$
K=\frac{\hbar v_{F}}{2 \pi a_{t 1} a_{t 2}}
$$

where $a_{t_{\nu}}$ are the interchain distances of the chains which run along the $\mathrm{z}^{-}$ direction. The Fermi velocities for the anisotropic metal are denoted $v_{F}, v_{t 1}$ and $v_{t 2}$, respectively. In terms of the Fourier component $V(\mathbf{Q})$ of the short range impurity potential ( $\mathbf{Q}$ denotes the CDW nesting vector) and in terms of the half gap $\Delta$, the electron density $n_{0}$, Fermi energy $\epsilon_{F}$, and electron-phonon coupling constant $\lambda_{Q}$ the pinning amplitude $V_{0}$ is given by $V_{0}=n_{0} V(Q) \Delta /\left(4 \lambda_{Q} \epsilon_{F}\right)$ [14]. $V_{0}$ has dimension of energy. We neglect dislocation lines in the CDW lattice and consider the phase to be unique over the crystal.

As in [3] we perform an isotropy scaling in the static CDW energy $E=\int d^{3} x \mathcal{H}$ according to $z^{\prime}=z, x^{\prime}=x v_{F} / v_{t 1}$, and $y^{\prime}=y v_{F} / v_{t 2}$ and use the old names. Then $\mathcal{H}$ becomes

$$
\mathcal{H}=\frac{1}{2} K(\nabla \varphi)^{2}+V_{0} \delta\left(\mathbf{x}-\mathbf{x}_{i}\right) \cos (\mathbf{Q} \mathbf{x}+\varphi)
$$

with $K=\hbar v_{t 1} v_{t 2} /\left(2 \pi a_{t 1} a_{t 2} v_{F}\right)$, while $V_{0}$ remains unchanged. The formal solution of the Poisson equation following from (3) gives divergent energy in three dimensions. We introduce a cutoff $\xi$ which is of the order of the amplitude coherence length $\hbar v_{F} / \Delta$ into our model by setting $\varphi\left(\left|\mathbf{x}-\mathbf{x}_{i}\right| \leq \xi / 2\right) \equiv \varphi_{i}$. Thus the phase in our quasi-isotropic setting is constant inside a sphere of diameter $\xi$ centered at the impurity. $\xi$ is a constant parameter of the model. This case corresponds to the following modified inhomogenity in the Poisson equation associated to (3):

$$
-V_{0} \delta\left(\left|\mathbf{x}-\mathbf{x}_{i}\right|-\xi / 2\right) \frac{\sin \left(\mathbf{Q} \mathbf{x}_{i}+\varphi_{i}\right)}{\pi \xi^{2}}
$$

For convenience we fix the phase at infinity by requiring $\varphi_{\infty}=0$. A phase $\varphi_{\infty} \neq 0$ can be trivially transformed away from all our equations, especially from the energies to be calculated. The latter, therefore, do not directly reflect pinning. Phase pinning, however, shows up in the pinning force [5, 13].

The solution of the modified Poisson equation for the phase is

$$
\varphi\left(\left|\mathbf{x}-\mathbf{x}_{i}\right| \geq \xi / 2\right)=\frac{\xi}{2} \frac{\varphi_{i}}{\left|\mathbf{x}-\mathbf{x}_{i}\right|}
$$

In (3) $\mathbf{Q} \mathbf{x}_{i}$ acts as a random phase uniformely distributed in $(-\pi, \pi)$ and we will denote it as $\Gamma$. By averaging the total energy over $\Gamma$ we obtain the CDW energy per pin of a random ensemble of local pins. 
The total CDW energy associated with the solution $\varphi(\mathbf{x})$ becomes $E=\pi K \xi \varphi_{i}^{2}+$ $V_{0} \cos \left(\Gamma+\varphi_{i}\right)$. With the pinning parameter

$$
h \equiv \frac{V_{0}}{2 \pi K \xi}
$$

the energy can be expressed as

$$
E=V_{0}\left(\frac{\varphi_{i}^{2}}{2 h}+\cos \left(\Gamma+\varphi_{i}\right)\right) .
$$

Finally the phase $\varphi_{i}$ follows from the requirement $\partial E / \partial \varphi_{i}=0$ or

$$
\varphi_{i}=h \sin \left(\Gamma+\varphi_{i}\right)
$$

This is an implicit equation for $\varphi_{i}$ in terms of $\Gamma$. Equation (8) also appears in the mean field model of Fisher [15] for the multivalued static solutions at $h>1$ in the presence of an external field.

Knowing $\varphi_{i}(\Gamma)$ the reduced averaged CDW energy $e(h)$ for example can be expressed as

$$
e(h) \equiv<E>/ V_{0}=\frac{1}{2 \pi} \int_{-\pi}^{\pi} d \Gamma\left\{\frac{h}{2} \sin ^{2}\left(\Gamma+\varphi_{i}(\Gamma)\right)+\cos \left(\Gamma+\varphi_{i}(\Gamma)\right)\right\} .
$$

It is noted that (8) can also be obtained without considering $\varphi_{i}$ as a variational parameter by starting from the inhomogeneous phase equation.

We summarize the principal features and assumptions of our model:

1. Three-dimensional Fukuyama-Lee-Rice model with one randomly placed impurity

2. Averaging over the impurity position amounts to an ensemble average over many local, i.e., independent pins. Weak pinning is excluded.

3. Neglect of quantum tunneling and thermal fluctuations. For a discussion of the corresponding conditions see [9]. It is also noted that $V_{0} \gg k_{B} T$ holds for strong pins.

The following two sections derive exact results from this model. 


\section{Average CDW Energy}

We begin by investigating the average CDW energy in the equilibrium state. This also serves as an introduction to our method of solution.

Our model is nontrivial because of equation (8) and its variety of solutions. For $h<1$ there exists one and only one solution $\varphi_{i}$ for every $\Gamma$. Writing (\&) as

$$
w=\Gamma+h \sin w ; \quad w \equiv \varphi_{i}+\Gamma,
$$

the solution $w(\Gamma)$ maps the interval $-\pi \leq \Gamma<\pi$ one to one onto $-\pi \leq w<\pi$. It is then easy to calculate $e(h \leq 1)$. Transforming the integration over $\Gamma$ in (9) to an integration over $w$ using $d \Gamma / d w=1-h \cos w$ gives immediately

$$
e(h \leq 1)=\frac{1}{2 \pi} \int_{-\pi}^{\pi} d w(1-h \cos w)\left[\frac{h}{2} \sin ^{2} w+\cos w\right]=-\frac{h}{4} .
$$

This argument fails for $h>1$ when more than one solution of (10) exists. For $h>1$ at least three solutions solutions exist. For $h=3$ the situation is depicted in Fig. 1. In general these solutions give different energies. The physical relevant solution is the one with the lowest energy. Choosing this solution for each $\Gamma$ defines the integration path.

Formally the average energy above the threshold can be expressed as

$$
e(h)=\frac{1}{2 \pi} \sum_{\nu}\left[\sin w-\frac{h}{4} w-\frac{3 h}{8} \sin 2 w-\frac{h^{2}}{6} \sin ^{3} w\right]_{w_{i}^{(\nu)}}^{w_{a}^{(\nu)}} .
$$

$\left(w_{i}^{(\nu)}, w_{a}^{(\nu)}\right)$ are the endpoints of appropriate integration intervals along the $w$-axis giving minimum energy. The endpoints of the path are $w_{i}^{(1)}=-\pi, w_{a}^{(1)}=-w_{A}$ and $w_{i}^{(2)}=w_{A}, w_{a}^{(2)}=\pi$ where $w_{a}=\sin \left(w_{A}\right)$, i.e., there is a jump from $-w_{A}$ to $w_{A}$ at $\Gamma=0$.

The value $h=1$ is a singular point in the following sense: The dependence of $e(h)+h / 4$ on $h$ changes taking on locally the form of a power law with exponent $b$. Eq. (12) can be used to calculate the exponent $b$ defined by

$$
e(h)+\frac{h}{4} \sim \epsilon^{b}, \quad \epsilon \equiv h-1 \ll 1 .
$$

The value of $w_{A}$ near $h=1$ can be found perturbatively. To lowest order with respect to $\epsilon$ it is $w_{A}=\sqrt{6 \epsilon}$. Eq. (12) then gives

$$
e(h)+\frac{h}{4}=\frac{8}{35 \pi} \sqrt{6} \epsilon^{7 / 2}
$$


i.e., the exponent is $b=7 / 2$. To complete the analytical investigations, the behaviour for $h \gg 1$ is studied. In this limit the value of $w_{A}$ is about $\pm \pi(1-1 / h)$ which gives

$$
e(h)=-1+\left(\frac{\pi^{2}}{6}-1\right) / h, \quad h \gg 1
$$

The usual strong pinning limit $\langle E\rangle_{s p}=-V_{0}$ is thus asymptotically approached albeit at a rather slow rate. It is also noted that for $\left|\arccos (1 / h)-\sqrt{h^{2}-1}\right|>\pi$ additional solutions exist which lead to more and more metastable states.

\section{Average Pinning Force}

Physically more important than the average energy $e(h)$ is the average pinning force the CDW experiences when it is moved adiabatically. This force is

$$
F(h)=-\frac{Q}{2 \pi} \int_{0}^{2 \pi} d \Gamma \frac{d E(w(\Gamma), \Gamma)}{d \Gamma} \equiv Q V_{0} f(h) .
$$

The pinning force $F(h)$ vanishes for $h \leq 1$ since $d E / d \Gamma$ then is a single valued and periodic function over the interval $-\pi \leq \Gamma<\pi$. Physically it means that the pinning forces from different local impurities cancel [5].

Above threshold transitions between branches of different energy occur. Using the energy profile

$$
e(w, \Gamma) \equiv \frac{E}{V_{0}}=\frac{(w-\Gamma)^{2}}{2 h}+\cos w
$$

and

$$
\frac{d e(w(\Gamma), \Gamma)}{d \Gamma}=-\frac{w-\Gamma}{h}=\sin w
$$

the reduced force $f(h)$ can be treated in anology to the energy. The result is

$$
f(h)=\frac{1}{2 \pi}\left[\frac{h}{4}\left(\cos 2 w_{B}-\cos 2 w_{c}\right)-\left(\cos w_{B}-\cos w_{c}\right)\right] .
$$

Here $w_{B}$ is the point of the energy minimum $e\left(w_{B}, \Gamma_{c}\right)$ into which a a transition occurs from the critical metastable state characterized by a horizontal inflection point in the energy profile at $\left(w_{c}, \Gamma_{c}\right)$. The r.h.s. of (19) is just the difference in energy of these two states devided by $2 \pi$. 
It is possible to give a more geometrical description of this process and the resulting force: Above threshold the force from shifts of the CDW by less than $x=\Gamma_{c} / Q$ where $\Gamma_{c}$ is given by $\Gamma_{c}=\sqrt{h^{2}-1}-w_{c}$ with $\left.w_{c}=\arccos (1 / h)\right)$ is elastic. Larger shifts populate metastable states.

For $\Gamma_{c}<\pi$ the averaging path is from $-\pi$ to $-w_{c}$ and then from $w_{B}$ to $\pi$ as shown in Fig. 1. It runs over the bifurcation point $\left(\Gamma_{c},-w_{c}\right)$ and jumps to the upper branch starting at $w_{B}=\Gamma_{c}+h \sin \left(w_{B}\right)$. This is clearly seen in the energy profile $e(w, \Gamma)$.

Near $h=1$ one can use the perturbative results $w_{c}=\sqrt{2 \epsilon}, w_{A}=2 w_{c}$ to find

$$
F(h)=\frac{9}{4 \pi} Q V_{0} \epsilon^{2}, \quad \epsilon \ll 1 .
$$

Thus pinning sets in at the threshold $h=1$ with exponent $\beta=2$.

For larger $h$ the situation is more complicated since more metastable states exist. The averaging path now extends over $n$ periods in order to sample all metastable states. The jump is from $\left(\Gamma_{c},-w_{c}\right)$ to the point $\left(\Gamma_{c}, w_{B}\right)$ which gives the lowest energy. $w_{B}$ is that value on the curve $\Gamma+h \sin (w)$ that lies nearest to $(2 n-1) \pi$ where $E(w)$ has its absolute minimum. The energy difference is $E\left(-w_{c}\right)-E\left(w_{B}\right)$ with $E\left( \pm w_{c}\right)=V_{0}(h / 2+1 /(2 h))$. Whenever $\Gamma_{c}$ crosses a

value $2 \pi n(n=1,2,3, \ldots)$, i.e., when $h=h_{n}$ where $h_{n}$ is found from $\sqrt{h_{n}^{2}-1}-$ $\arccos \left(1 / h_{n}\right)=2 n \pi$ (approximate solution $h_{n}=2 n \pi+\pi / 2$ ) the winding number changes from $n$ to $n+1$ and $f(h)$ is reduced correspondingly. This leads to a saw-toothed structure of $f\left(h_{n-1}<h \leq h_{n}\right)=\left(h / 2+1 /(2 h)-E\left(w_{B}\right)\right) /(2 \pi n)$ $\left(h_{0} \equiv 1\right)$. Thus $f(h)$ oscillates around the asymptotic value $1 / 2$ with approximate period $2 \pi$ and decreasing peak to peak amplitude $\delta f\left(h_{n}\right)=f\left(h_{n}\right) /(n+1)$. Fig. 2 displays the reduced pinning force $f(h)$. It is noted that the unscaled force (16) contains the pinning amplitude $V_{0}$ and does not saturate for $h \rightarrow \infty$ as in Larkin's model [9].

The threshold behaviour of the pinning force is also found in the one-dimensional pinning model in 9, 13 and is a general feature of local impurity phase pinning [5].

\subsection{Pinning Parameters}

The relation of the pinning parameter $h$ to the usual measure of pinning strength will now be discussed. According to (7) and taking the isotropy scaling into account, $h$ is given by:

$$
h=V_{0} \frac{a_{t 1} a_{t 2} v_{F}}{v_{t 1} v_{t 2} \hbar \xi}
$$

The standard measure [16 for impurity energy to elastic energy in an anisotropic three-dimensional CDW continuum is 


$$
\epsilon_{i}=2 \pi V_{0} a_{t 1} a_{t 2} c_{3}^{1 / 3}\left(\frac{v_{F}^{2}}{v_{t 1} v_{t 2}}\right)^{2 / 3} \frac{1}{\hbar v_{F}}
$$

where $c_{3}$ is the impurity concentration. The effective distance between pins is $\ell=\left(c_{3} v_{t 1} v_{t 2} / v_{F}^{2}\right)^{-1 / 3}$. Thus $\epsilon_{i}$ is related to $h$ by

$$
\epsilon_{i}=2 \pi \xi h / \ell
$$

The applicability of the theory requires $\xi$ to be smaller than $\ell$. Using the values $v_{F}=5 \cdot 10^{7} \mathrm{cms}^{-1} \approx 10 v_{t}, \Delta=1000 k_{B} T$, and an impurity concentration of one ppm the ratio $\xi / \ell$ is estimated as 0.01 One can, nevertheless, conclude that the strong pinning limit in our model, $h \gg 1$, also requires large $\epsilon_{i}$. Weak pinning occurs for $\epsilon_{i}<1$. The region $0<h \leq 1$ where local pinning is absent thus is concealed by weak pinning which is ubiquitous in less than four dimensions.

\section{$5 \quad$ Descreening}

So far we have considered the case of full screening: There are enough thermally excited quasiparticles (or normal carriers as in $\mathrm{NbSe}_{3}$ ) to completely screen out the Coulomb forces between charge fluctuations associated with phase deformations. It is known that descreening stiffens the CDW. This stiffening of the CDW leads to a corresponding increase of the phason velocity which has been observed by neutron scattering [17] and explained in [18] as a descreening effect. In a simple approximation involving only the condensate fraction $N<1$ one can define an effective stiffness constant

$$
K_{e f f}=\frac{K}{1-N}
$$

to take care of descreening within the elastic CDW model [19, 20, 21, 22, 10, 23, 24]. Following [24] this is a reasonable approximation for $1-N>\zeta \equiv \epsilon_{t} \hbar v_{F} /\left(8 e_{0}^{2}\right)$ $\left(\epsilon_{t}\right.$ : static transverse dielectric constant). In the opposite limit Coulomb interactions require a different approach. The modified stiffness constant changes the pinning parameter $h$ according to

$$
h \rightarrow h \sqrt{1-N} \equiv h_{e f f}
$$

since a scaling in chain direction becomes necessary to maintain quasi isotropy.

Our earlier formulae hold with this replacement. When $h_{\text {eff }}$ becomes less than unity in decreasing the temperature local impurity phase pinning stops. The 
singularity in the local pinning force at $h_{e f f}=1$ is likely to be masked by thermal fluctuations and by weak pinning.

The fact that local pinning centers require a minimum strength to act as strong pins is possibly the reason for the following observation in [25]: The CDW in Ti doped $\mathrm{NbSe}_{3}$ which is fully screened due to a partially gapped Fermi surface exhibits weak instead of the expected strong pinning.

\section{Relation to Larkins's model}

We want to point out the similarities and differences in our approach to Larkin's model [9] and fill in some additional information about it.

Larkin uses a single chain model [26] with one impurity at $x=x_{i}$. It is defined by the energy functional:

$$
E=\frac{E_{s}}{8} \int d x\left[1-\cos \varphi(x)+\frac{1}{2}\left(\frac{d \varphi(x)}{d x}\right)^{2}\right]+V_{0}\left[1-\cos \left(\Gamma+\varphi\left(x_{i}\right)\right)\right] .
$$

In (26) the length $x$ is scaled to $\hbar v_{F} /\left(2 t_{\perp}\right)$. The interchain coupling energy is denoted by $t_{\perp}$ and $E_{s}=8 t_{\perp} / \pi$ is the energy of a $2 \pi$ phase soliton [27] ( $E_{s}$ is called $w$ in [9]).

¿From the general theory in [24] which describes the CDW as a system of coupled chains Larkin's model follows under three assumptions:

1. Phases on neighbouring chains are set to zero which turns the interchain coupling into the Sine-Gordon type self interaction $1-\cos \varphi$ in (26). Thus phases on different chains are independent. In contrast the phase varies slowly across the chains in our model, a case more appropriate to a screened situation.

2. The low temperature or descreened limit is understood when the quasiparticle fraction $1-N$ is smaller than the Coulomb coupling constant $\zeta$.

3. The value $\zeta$ of the Coulomb coupling constant mentioned in Sec. 5 is fixed at $1 / 8$. In reality a smaller value holds because of $v_{F}=O\left(10^{7} \mathrm{cms}^{-1}\right)$.

The model (26) has uncharged dipole solutions for which the phase $\varphi_{i}=\varphi\left(x_{i}\right)$ at the impurity obeys the matching condition $\left(h \equiv 4 V_{0} / E_{s}\right)$

$$
-2 \sin \frac{\varphi_{i}}{2}=h \sin w
$$

This equation replaces our equation (10). For any solution $\varphi_{i}^{(1)}$ it has anotherusually inequivalent-solution $\varphi_{i}^{(2)}$ with $\varphi_{i}^{(2)}=2 \pi-\varphi_{i}^{(1)}$. The elastic energies are 
$E_{s}\left[1-\cos \left(\varphi_{i}^{(1)} / 2\right)\right]$ and $E_{s}\left[1-\cos \left(\varphi_{i}^{(2)} / 2\right)\right]=E_{s}\left[1+\cos \left(\varphi_{i}^{(1)} / 2\right)\right]$. We consider here the domains $0 \leq w<2 \pi$ and $0 \leq \varphi_{i}<2 \pi$. The model is thus characterized by one metastable state.

The mechanism of pinning force generation for $h<2$ is precisely the same as discussed in Sec. 4: Transition from a metastable state with relative energy $\Delta E$ which becomes a horizontal inflection point in the energy profile $E(w, \Gamma)$ for $\Gamma=\Gamma_{c}$ down to the ground state. The average pinning force is then:

$$
F=\frac{Q \Delta E}{2 \pi}
$$

In our model this mechanism prevails for all $h>1$ and more and more metastable states appear for increasing $h$. In Larkin's model no horizontal inflections points exist for $h>2$. Instead two $2 \pi$-solitons are created when $\varphi_{i}$ changes by $2 \pi$ in a corresponding change of $\Gamma$. This leads to $F(h>2)=Q E_{s} / \pi$ in [9] while the average value of $F(h)$ increases linearily with $h$ in our model as implied by the strong pinning concept.

It is possible to study the special case $h=2$ analytically because the exact solution of (27) - expressed as $w=w(\Gamma)$ - is available:

$$
w=\frac{\Gamma}{3}+\frac{4 \pi}{3} n, \quad n=0,1 ; \quad w=-\Gamma+2 \pi .
$$

From the energy profile

$$
e(w, \Gamma)=\frac{E}{E_{s}}=1-\cos \frac{w-\Gamma}{2}+\frac{h}{2} \sin ^{2} \frac{w}{2},
$$

and the matching condition (27) one finds

$$
\frac{d e(w(\Gamma), \Gamma)}{d \Gamma}=\frac{h}{4} \sin w=-\frac{1}{2} \sin \frac{w-\Gamma}{2} .
$$

The inflection points for $h=2$ are $\left(\Gamma_{c}=3 \pi / 2, w_{c}=\pi / 2\right),\left(\Gamma_{c}=\pi / 2, w_{c}=3 \pi / 2\right)$ and a corresponding ground state is $\left(\Gamma_{c}=3 \pi / 2, w_{B}=11 \pi / 6\right)$. Thus one finds

$$
F(h=2-0)=\frac{Q E_{s}}{2 \pi}\left[e\left(w_{c}, \Gamma_{c}\right)-e\left(w_{B}, \Gamma_{c}\right)\right]=\frac{Q E_{s}}{4 \pi} \cos \frac{\pi}{6}=\frac{3 \sqrt{3}}{8 \pi} Q E_{s} .
$$

For $h=2+0$ the path to follow in the integral

$$
F=-\frac{Q E_{s}}{2 \pi} \int_{0}^{2 \pi} d \Gamma \frac{d e}{d \Gamma}=-\frac{Q E_{s}}{8 \pi} h \int_{0}^{2 \pi} d \Gamma \sin w(\Gamma)
$$


is continuous and goes from $w(0)=2 \pi$ to $w\left(\Gamma_{c}\right)=3 \pi / 2$ and then back to $w(2 \pi)=2 \pi$ resulting in a phase change of $\Delta \varphi_{i}=-2 \pi$. The integration gives

$$
F(h=2+0)=\frac{Q E_{s}}{\pi} .
$$

This is the value of the force for all $h>2$ as pointed out in [9].

Further analytical results are found for $h<2$. From the topological condition $d \Gamma / d w=0$ of the critical inflection point one gets

$$
e\left(w_{c}, \Gamma_{c}\right)=1+\frac{h}{4}-\frac{3}{4} \sqrt{\frac{4-h}{4}}
$$

with

$$
w_{c}=\arccos \sqrt{\frac{4-h}{3 h^{2}}}, \quad \Gamma_{c}=\arccos \sqrt{\frac{4-h}{3 h^{2}}}+2 \arccos \sqrt{\frac{n^{2}-1}{3}} .
$$

The perturbative expansion of the force $F(h)$ slightly below $h=2$ then leads to

$$
F(h)=F(h=2-0)-\frac{Q E_{s}}{4 \pi}\left\{\sqrt{3}+\frac{1}{2} \sqrt{\frac{1}{3}}\right\} \sqrt{2-h}+O(2-h) .
$$

It is clear that the threshold for pinning is $h=1$ because there are no metastable states for $h<1$. The intermediate regime $1<h<2$ is treated numerically and the result is shown in Fig. 3. The behaviour near $h=1$ is again $F(h) \sim$ $(h-1)^{2}=\epsilon^{2}$. The critical exponent is thus $\beta=2$ which seems to be universal for local pinning.

\section{Discussion}

From its very definition local impurity pinning is expected to be independent of spatial dimensionality $d$. This is born out by our approach. Repeating the calculations of Sec. 2 for $d=2$ and $d=1$ always leads to the central equation (10). However, the pinning parameter $h \equiv h_{d}$ is not any more given by (6) which refers to $d=3$. Using half the the mean distance $\ell_{d} \gg \xi$ between impurities as the distance from the impurity where $\varphi$ vanishes it is found that $h_{2}=V_{0} a_{t} \ln \left(\ell_{2} / \xi\right) /\left(\hbar v_{t}\right)$ for $d=2$. For $d=1 h_{1}=\pi V_{0} \ell_{1} /\left(2 \hbar v_{F}\right)$ is obtained. The relation (23) between the pinning parameter $h_{d}$ and $\epsilon_{i}$ becomes $\epsilon_{i}=2 \pi h_{2} / \ln \left(\ell_{2} / \xi\right)$ for $d=2$ and $\epsilon_{i}=4 h_{1}$ for $d=1$. These relations are similar to (23) and do not change the 
conclusions significantly. The case $d=1$ which does not require the short distance cut-off $\xi$ is unrealistic since real CDW are never one-dimensional.

In summary we have studied a solvable model of local impurity phase pinning which realizes the local pinning scenario in [5], namely singular points, threshold behaviour, and metastable states. In contrast to pinning in type II superconductors static descreening and the possible deactivation of local pins at low temperatures are unique to semiconducting CDW (and spin density) systems. These results have been obtained within a phase only model plus some amendments for descreening. Especially at low temperatures more general models, e.g., those in [24] which take nonlinear screening (band bending) into account may be considered.

The authors thank P.B. Littlewood who initiated this study. They also thank S.N. Artemenko for helpful discussions. 


\section{References}

[1] G. Grüner, Rev. Mod. Phys. 60, 1129 (1988).

[2] H. Fukuyama, P.A. Lee, Phys. Rev. B 17, 535 (1978).

[3] P.A. Lee, T.M. Rice, Phys. Rev. B 19, 3970 (1979).

[4] A.I. Larkin, Yu.N. Ovchinnikov, Zh. Eksp. Theo Fiz. 65, 1704 (1973) (Sov. Phys. JETP 38, 854 (1974)).

[5] A.I. Larkin, Yu.N. Ovchinnikov, J. Low. Temp. Phys. 34, 409 (1979).

[6] S. Abe, J. Phys. Soc. Jpn. 55, 1987 (1986).

[7] N.I. Lebedev, A.P. Levanyuk, A.S. Sigov, Zh. Eksp. Teor. Fiz. 92, 248 (1987) (Sov. Phys. JETP 65, 140 (1987)).

[8] J.R. Tucker, W.G. Lyons, G. Gammie, Phys. Rev. B 38, 1148 (1988).

[9] A.I. Larkin, Zh. Eksp. Teor. Fiz. 105, 1793 (1994) (JETP 78, 971 (1994)).

[10] A.I. Larkin, S. Brazovskii, Solid State Comm. 93, 275 (1995).

[11] S. Brazovskii, in: Physics and Chemistry of Low-Dimensional Inorganic Conductors, eds. C. Schlenker et al., Plenum Press, New York, 1996.

[12] S. Brazovskii, A. Larkin, Synth. Met. 86, 2223 (1997).

[13] Yu.N. Ovchinnikov, K. Biljakovich, J.C. Lasjaunias, P. Monceau, Europhys. Lett. 34, 645 (1996).

[14] S.N. Artemenko, A.N. Kruglov, Zh. Eksp. Theo. Fiz. 83, 1134 (1982) (Sov. Phys. JETP 56, 643 (1982)).

[15] D. S. Fisher, Phys. Rev. B 31, 1396 (1985).

[16] H. Matsukawa, H. Takayama, J. Phys. Soc. Jpn. 56, 1507 (1987).

[17] B. Hennion, J.P. Pouget, M. Sato, Phys. Rev. Lett. 68, 2374 (1992).

[18] A. Virosztek, K. Maki, Phys. Rev. Lett. 69, 3265 (1992).

[19] Y. Kurihara, J. Phys. Soc. Jpn. 49, 852 (1980).

[20] K.Y.M. Wong, S. Takada, Phys. Rev. B 36, 5476 (1987).

[21] S.N. Artemenko, A.F. Volkov, Synth. Met. 29, F 407 (1989).

[22] A. Virosztek, K. Maki, Phys. Rev. B48, 1368 (1993). 
[23] S.N. Artemenko, W. Wonneberger, J. Phys. I France 6, 2079 (1996).

[24] S.N. Artemenko, Zh. Eksp. Theo. Fiz. 111, 1494 (1997) (JETP 84, 823 (1997)).

[25] J. McCarten, D.A. DiCarlo, M.P. Maher, T.L. Adelman, R.E. Thorne, Phys. Rev. B46, 4456 (1992).

[26] S. Brazovskii, in: Charge Density Waves in Solids, eds. L. Gorkov, G. Grüner, Elsevier, Amsterdam, 1990.

[27] M.J. Rice, A.R. Bishop, J.A. Krumhansl, S.E. Trullinger, Phys. Rev. Lett B36, 432 (1976). 


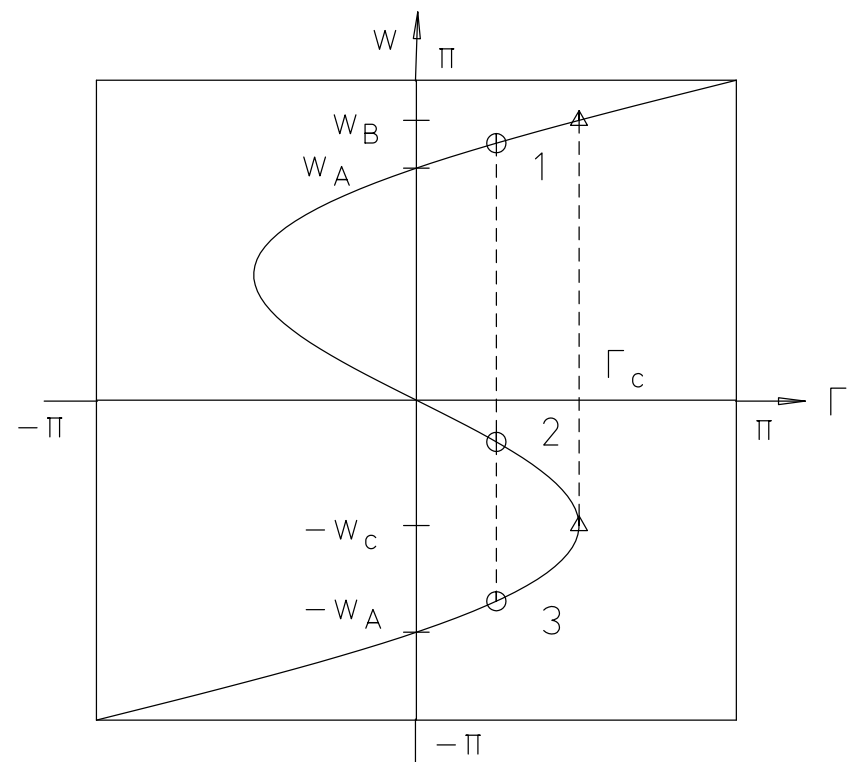

Figure 1: Display of the solutions of the self consistency equation (10) in the form $w=\Gamma+h \sin w$ for $h=3$, i.e., not too far above the pinning threshold $h=1$. The circles 1, 2, 3 indicate the solutions for $-\Gamma_{c}<\Gamma \leq \Gamma_{c} \cdot\left(\Gamma_{c},-w_{c}\right)$ is bifurcation point. The pinning force results from the vertical transition from $-w_{c}$ to $w_{B}$ on the upper branch. 


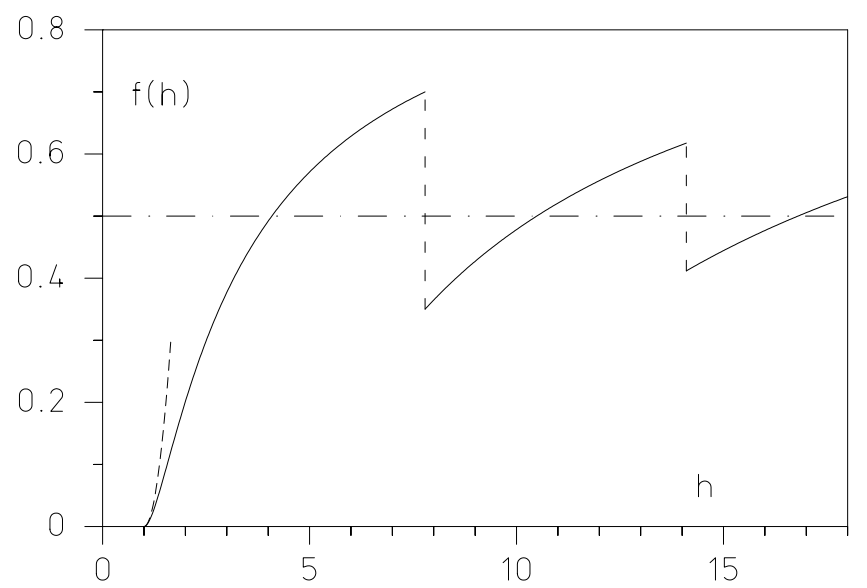

Figure 2: Reduced pinning force $f$ in units of $Q V_{0}$ as function of pinning parameter $h$. The local impurity phase pinning force $f$ sets in at $h=1$ with exponent $\beta=2$. Saturation value $f(h \rightarrow \infty)=1 / 2$, the quadratic treshold behaviour, and the jumps at the points $h_{n} \approx 2 \pi n+\pi / 2, n=1,2, \ldots$ are indicated by broken lines.

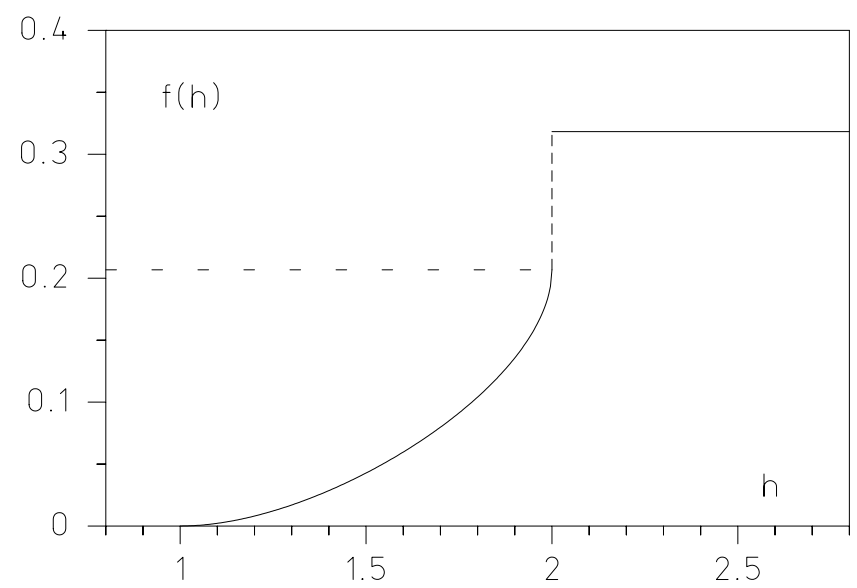

Figure 3: Reduced pinning force $f(h)$ in units of $Q E_{s}$ as function of pinning parameter $h$ for Larkin's model. There is no pinning force below $h=1$ and $f(h)$ jumps from $3 \sqrt{3} /(8 \pi)$ to the final value $1 / \pi$ at $h=2$. Note the different normalization of $f(h)$ in comparison to Fig. 2. 